\title{
El impuesto a la plusvalía y su impacto en el sector de la construcción en el cantón Ambato, provincia de Tungurahua
}

\author{
URL: http://revistas.uta.edu.ec/erevista/index.php/bcoyu/article/view/686 DOI: http://dx.doi.org/10.31164/bcoyu.20.2019.686
}

\author{
Edgar Mera - Bozano'; César Salazar - Mejía; Alex Solís - Gavilanes ${ }^{3}$ \\ Fecha de recepción: 7 de octubre de $2018 \quad$ Fecha de aceptación: 7 de diciembre de 2018
}

\section{Resumen}

El estudio establece la afectación de la Ley de plusvalía para evitar la especulación de la Tierra en el desempeño del sector de la construcción del Cantón Ambato; la desinformación generada por los medios de comunicación y las cámaras de la construcción atribuyó a la normativa el decrecimiento económico en el sector, en apenas un año desde su publicación, de ahí su derogatoria en el año 2018 mediante consulta popular; sin embargo analizados los factores se concluye que no fue la vigencia de la Ley la causante de la recesión del sector sino otros factores macro-económicos. Dado el enfoque de la investigación se acudió a fuentes de información de las bases de datos oficiales de Instituciones estatales relacionadas.

Palabras clave: Tributo, impuesto, especulación, empleo, rentabilidad.

\section{Abstract}

The study establishes the affectation of the Law of surplus value to avoid the speculation of the Earth in the performance of the sector of the construction of Canton Ambato; the misinformation generated by the media and the chambers of construction attributed to the regulations the economic decline in the sector, in just one year since its publication, hence its repeal in the year 2018 through popular consultation; However, after analyzing the factors, it is concluded that it was not the Law that caused the recession of the sector but other macroeconomic factors. Given the focus of the research, sources of information were accessed from the official databases of related state institutions.

Keywords: Tribute, tax, speculation, employment, profitability.

\section{Introducción}

En Ecuador el llamado impuesto a la "plusvalía" aprobado en diciembre de 2016 busca evitar la especulación de la tierra, conforme su estructura el impuesto grava el incremento del valor de los bienes inmuebles (Asamblea Nacional República del Ecuador, 2016) y la utilidad extraordinaria que se genere como resultado de la segunda venta de estos bienes; sin embargo, el impuesto recibió fuertes críticas, particularmente de las cámaras de la industria de la construcción, bajo el supuesto no comprobado de que "una medida de esta naturaleza desalentará al inversionista inmobiliario", tema que se hizo eco en los medios de comunicación, que sin información alguna patrocinaron lo "perjudicial" del tributo (La Hora, 2016).

El presente estudio tiene como objetivo determinar el impacto del impuesto a la plusvalía en el sector de la construcción en el cantón Ambato provincia de Tungurahua por el período de su vigencia, a través del análisis de la estructura del impuesto y de la evaluación de los factores que intervinieron en la economía para la recesión del sector de la construcción, para ello, es necesario analizar los aspectos normativos que contiene la Ley Orgánica para Evitar la Especulación sobre el Valor de las Tierras que al parecer, previo a la consulta popular de 2018 no fue socializado de manera adecuada, además establecer los factores determinantes en la rentabilidad del sector de la construcción.

El impuesto fue discutido principalmente por la alta tasa marginal ya que este gravaba una tarifa del $75 \%$ sobre el monto en exceso adicional a la ganancia ordinaria, lo cual según ciertos sectores ha ocasionado que sus ingresos se reduzcan afectando el normal desarrollo de sus actividades.
En palabras de Correa (2017) la ley crea un país menos inequitativo, al permitir recuperar la inversión pública, captar las ganancias extraordinarias para la ciudad, e impedir la especulación y muchas veces muy injusta transferencia de valor de los compradores a los propietarios.

"Plusvalía" es el incremento del valor del suelo, sin modificar sus características, por acción de agentes económicos o circunstancias ajenas al respectivo propietario. Más sencillamente, la ganancia no generada por el esfuerzo del dueño del terreno (Correa Delgado, 2017).

Para el Servicio de Rentas Internas ente administrador de tributos internos en el Ecuador históricamente, reducidos segmentos de la sociedad han obtenido ganancias extraordinarias ilegítimas provenientes de la especulación en la tenencia de bienes inmuebles. Asimismo, de forma generalizada, los propietarios de los bienes inmuebles han ido aprovechando su incremento, ocasionado entre otros motivos, por la intervención del Estado a través de diferentes obras.

La ley evitará que los especuladores lucren con el sueño de los ciudadanos de tener casa en condiciones dignas, desincentivando la ganancia ilegítima generada a través de la obra pública, principalmente del acceso a información privilegiada. De esta manera se controlará la evasión de impuestos mediante la subvaloración de los predios en los catastros.

Se beneficiará a todos los ecuatorianos al evitar la especulación de la tierra, pero sobre todo al $36 \%$ de hogares en el país que no tienen vivienda propia y que desean acceder a una digna con un precio justo, permitiendo así el acceso equitativo a los bienes inmuebles (Servicio de Rentas Internas, 2016).

El estudio busca establecer si el impuesto regulaba la especulación de la tierra y si su aplicación podía evitarla, o en su defecto la creación del tributo ha sido un determinante para la recesión del sector de la construcción y su baja rentabilidad, aun cuando es conocido que organizaciones con gran acumulación de capital, invierten en la adquisición de tierras en lugares en donde existe la tendencia de que a un futuro inmediato el precio suba por mejoras en el sector que en ocasiones proviene de la obra pública (Ramírez Carreño, 2016).

Para Quintana (2010) en su estudio sobre la especulación menciona que lamenta desilusionar a quien piensa que la especulación podría erradicarse para dejar sólo "al libre mercado bueno". La existencia de especulación es indispensable si se quiere que haya competencia. En el estudio "pega especulación a los bienes raíces", indica que la especulación crea falsas expectativas en los compradores y vendedores, sólo beneficiando a los mismos especuladores (Garza R. , 2017).

Aunque no se pueda afirmar que se ha presentado una burbuja especulativa espacial, (Garza N. F., 2005) concluye que el crecimiento del precio del suelo durante el período fue exagerado y requiere de un análisis cuidadoso.

\section{Metodología}

El estudio tiene un enfoque cuali - cuantitativo, se trabajó con investigación secundaria disponible en bases de datos e informes que permitió establecer el impacto del impuesto en el sector de la construcción en su período de vigencia, ha sido necesario recoger información de los distintos entes de gobierno relacionados con la problemática y que manejan la información, particularmente la administración tribu- 
taria central y seccional, así como de ciertas entidades crediticias involucradas en la concesión de créditos hipotecarios necesarios para la construcción, y la información de las variaciones que presentan las estadísticas del INEC, información relacionada al período 2017. Con información del GAD Municipalidad de Ambato, se validó la evolución de las utilidades generadas en el sector en el período impositivo 2015 a 2017 así como el crecimiento del ingreso y la participación en la recaudación.

De la información del Servicio de Rentas Internas (SRI), se validó si el sector de la construcción que se contrajo en la economía fue aquella que corresponde a la venta de bienes inmuebles o a la que corresponde a la inversión pública, la tendencia creciente o decreciente de sus activos, pasivos y patrimonio, así como también la evolución de los ingresos, costos y gastos del sector con la finalidad de validar la afectación en la rentabilidad.

Del Banco del Instituto Ecuatoriano de Seguridad Social (BIESS) se analizó el crecimiento de los créditos hipotecarios, validando el comportamiento de las solicitudes y otorgamientos de créditos hipotecarios y el análisis de su reducción o incremento.

En cambio del Instituto Nacional de Estadística y Censos (INEC) es necesario validar la información que se refiere a la tendencia que ha tenido la contratación de la carga laboral en el sector en el periodo de vigencia de la plusvalía, y si a éste se le puede atribuir algún tipo de decrecimiento.

\section{Resultados}

A continuación, con información de la administración tributaria seccional se presenta la recaudación por concepto de las utilidades de tres períodos impositivos:

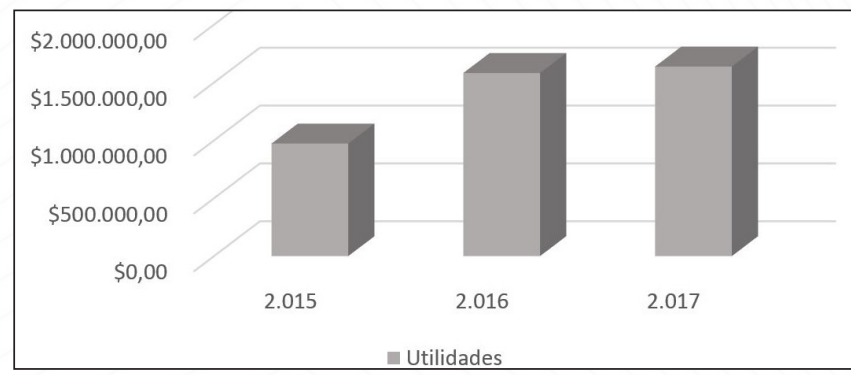

Fuente: Elaboración propia a partir del trabajo expuesto por elGobierno Autónomo Descentralizado Municipalidad de Ambato GAD (2017) Figura 1. Participación de recaudación en dólares

Analizado la evolución que han tenido los impuestos relacionados a la transferencia de inmuebles vemos para el año 2017, periodo en el cual se creó una nueva metodología para el cobro del llamado impuesto de la plusvalía, no ha tenido disminución relevante que se le pueda atribuir a la ley de la plusvalía, pues en el tema de utilidades ha sido creciente sin haber existido cambios de tarifas, utilidades generadas en la venta de inmuebles.

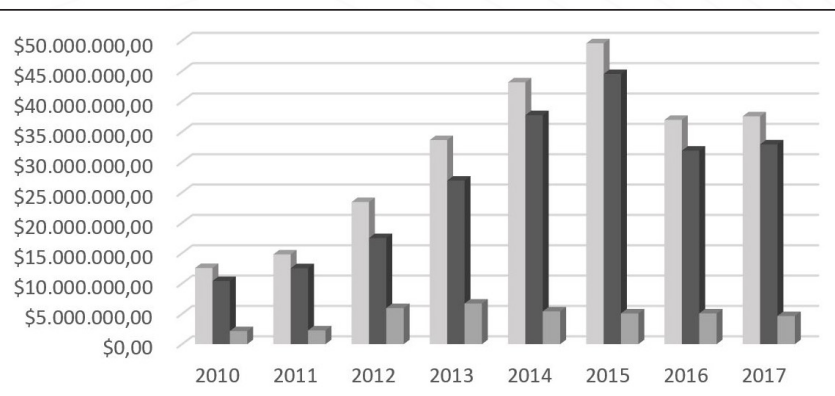

$\square$ TOTAL DEL ACTIVO $\quad$-TOTAL DEL PASIVO $\quad$ TOTAL PATRIMONIO NETO

Fuente: Elaboración propia a partir del trabajo expuesto por el Servicio de Rentas Intemas SRI (2018) Figura 2. Estado de Situación Financiera en dólares del sector de la construcción del cantón Ambato
De acuerdo a la información que proporciona el Servicio de Rentas Internas (SRI), el sector de la construcción del cantón Ambato presenta una tendencia creciente en sus activos hasta el año 2015, en el año 2016 existe una variación negativa de -25\% en relación al periodo anterior y en el año 2017 se recupera levemente con una variación del 2\%, el 2016 fue un año de recesión en el cual el Ecuador tuvo que soportar la baja del precio de Petróleo y un desastre Natural, ya en el 2017 se nota una leve recuperación.

Los pasivos muestran sintonía con los activos en los periodos de crecimiento 2015 y decrecimiento en el 2016, en el periodo 2017 hay un incremento del $3 \%$, se entendería que el sector genera deuda para cumplir con sus obligaciones con proveedores o para generar mayor productividad, alcanzando los 32 millones de dólares en Pasivos, frente a los casi 40 millones de Activos.

Las variaciones del Patrimonio neto del sector no muestran una caída significativa en el periodo de aplicación del impuesto, adicionalmente es necesario considerar que la inversión pública cayó y entre ello, el sector de la construcción, de tal forma que no necesariamente habría que atribuirle al impuesto la totalidad de las mínimas variaciones negativas.

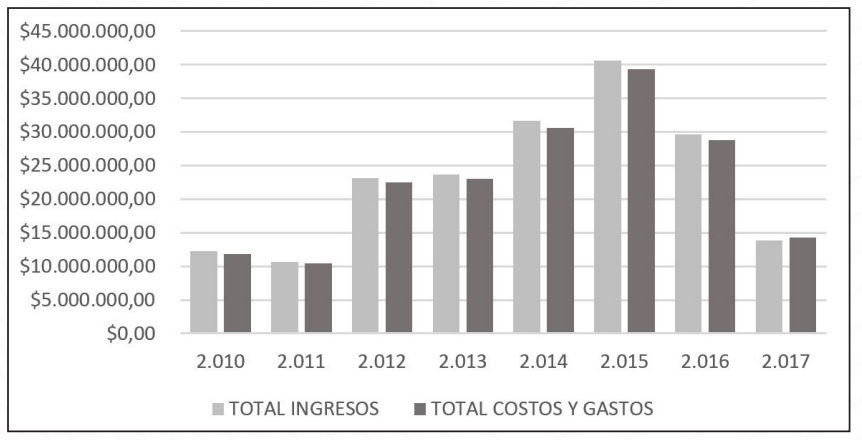

Fuente: Elaboración propia a partir del trabajo expuesto por el Servicios de Rentas Internas SRI (2018) Figura 3. Estado de Resultados en dólares del sector de la construcción del cantón Ambato.

El sector de la construcción en ventas o total de ingresos presenta variaciones tanto positivas como negativas en los últimos períodos, en el año 2011 existe una variación de -14\%, en el año 2012 se incrementa en 118\%, en el 2013 se incrementa el 2\%, en el año 2014 aumenta $34 \%$, con el $28 \%$ de incremento en el año 2015, en cambio en el año 2016 y 2017 se reduce en $-27 \%$ y $-53 \%$ respectivamente, sin embargo los ingresos en 2017 no han llegado a los niveles del 2010, los años que muestran mayores ingresos se relaciona con la inversión pública que ha realizado el Estado de acuerdo con mundoconstructor.com.ec (2018) el principal elemento que impactará en el escenario económico de este año es una caída en la formación bruta de capital fijo (inversión en construcción, maquinaria, equipo, etc.), las tendencias negativas se muestran desde 2016, periodo en el cual no se aplicaba el impuesto.

El total de costos y gastos del sector de la construcción presenta tendencias crecientes desde el año 2012 hasta el 2015, en el año 2016 se reducen los costos y gastos registrando una variación de - $27 \%$ y en el año 2017 el -50\%, los decrecimientos en el nivel del gasto ocurren desde el período 2016, período de recesión de la economía producto de factores anteriormente analizados.

En relación a las utilidades éstas muestran tendencias positivas en los períodos en los cuales el Estado destinó recursos a inversión pública, en el año de aplicación del impuesto no debería incidir pues el aspecto material del impuesto, la configuración del hecho generador se daba a la segunda venta.

El sector si bien muestra un decrecimiento, éste está fundamentado en la disminución de la inversión pública. De las 18 industrias que clasifica el Banco Central del Ecuador (BCE), 10 decrecieron en el 2016, mostrando a la construcción como una de las 5 industrias que más decrecieron en $-8,9 \%$, esta se ha venido contrayendo desde el 2014 , afectada por la caída de la inversión pública, salvaguardia, y no nece- 
sariamente por la aplicación de la Ley de Plusvalía, pues no se puede analizar la afectación de un tributo en apenas un año de su aplicación (Camara de Comercio de Guayaquil, 2017).

Tabla 1. Préstamos hipotecarios (USD millones) otorgados al sector de la construcción

\begin{tabular}{rrrrr}
\hline AÑO & $\begin{array}{c}\text { Entidades } \\
\text { Financieras }\end{array}$ & VARIACIÓN & BIESS & VARIACIÓN \\
\hline 2012 & $\$ 905,78$ & & $\$ 720,84$ & \\
2013 & $\$ 1.101,09$ & $22 \%$ & $\$ 554,58$ & $-23 \%$ \\
2014 & $\$ 1.190,58$ & $8 \%$ & $\$ 674,11$ & $22 \%$ \\
2015 & $\$ 1.174,66$ & $-1 \%$ & $\$ 685,01$ & $2 \%$ \\
2016 & $\$ 939,82$ & $-20 \%$ & $\$ 574,82$ & $-16 \%$ \\
2017 & $\$ 331,44$ & $-65 \%$ & $\$ 417,83$ & $-27 \%$
\end{tabular}

Fuente: Elaboración propia a partir del Banco del Instituto Ecuatoriano de Seguridad Social BIESS (2018)

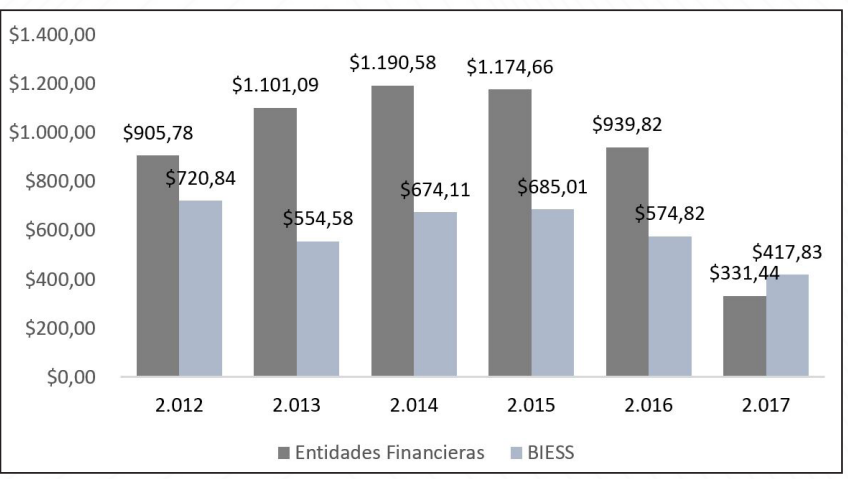

Fuente: Elaboración propia a partir del trabajo expuesto por el Banco del Instituto Ecuatoriano de Seguridad Social BIESS (2018) Figura 4. Préstamos hipotecarios (USD millones)

Los créditos hipotecarios otorgados tanto por instituciones financieras privadas como por el Banco del Instituto Ecuatoriano de Seguridad Social BIESS muestran una disminución de los montos otorgados durante el año 2016 y 2017. En las entidades financieras registran una reducción del -20\% en el año 2016 y en el 2017 de -65\%, por otro lado, el BIESS también registra una disminución en el año 2016 de $-16 \%$ y en el 2017 con $-27 \%$, al considerarse el impuesto a la segunda venta y visto que la tendencia de la adquisición de inmuebles se refiere a casas nuevas, no existe afectación por el impuesto, pues este no está diseñado para la venta de inmuebles sino hasta la segunda venta, se descarta que quienes adquirieron sus viviendas en años pasados sufran la consecuencia del impuesto pues al venderlas se configuraría una primera venta, y si fuera del caso que quien la compra la volviera a vender conforme la estructura del impuesto aplica una tasa de interés de aproximadamente el 8,6\% por año que mide el valor del dinero en el tiempo a lo que hay que sumarle la franja exenta, es decir la utilidad debería ser demasiado alta para que se llegue a tributar, y que justamente es lo que evita el impuesto, que se especule con el valor de la tierra.

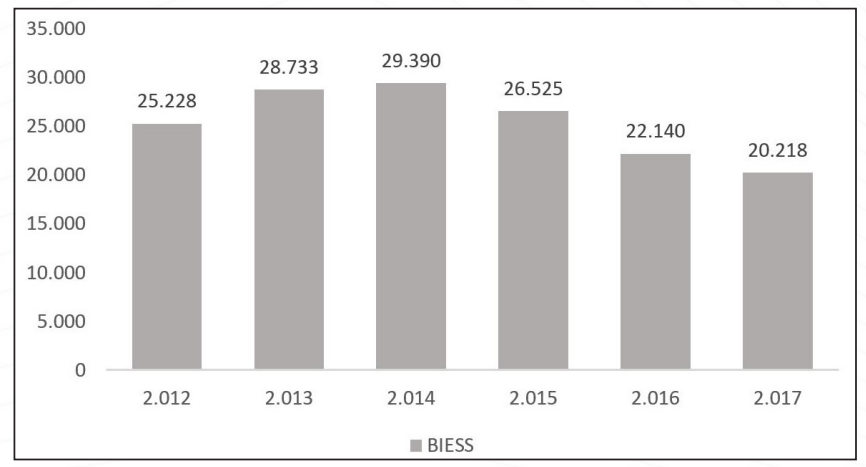

Fuente: Elaboración propia a partir del trabajo expuesto por el Banco del Instituto Ecuatoriano de Seguridad Social BIESS (2018) Figura 5. Préstamos hipotecarios (Número de operaciones)
Estos datos se pueden contrastar con el número de operaciones netas realizadas por el BIESS, ya que se observa van disminuyendo desde el año 2015 que registra 26.525 operaciones con una variación de -10\%, en el año 2016 registra 22.140 operaciones, una variación de -17\%, y en el año 2017 se registraron 20.218 operaciones con una variación de $-9 \%$.

La reducción de los créditos se configura por la falta de capacidad para el pago, se sufrió una recesión que pasó factura a varios sectores, se apreció el dólar lo que generó que el Estado tome medidas como la inclusión de salvaguardas, eso frenó al sector dentro de la economía sin que se distinga en aquello la aplicación del impuesto a la plusvalía.

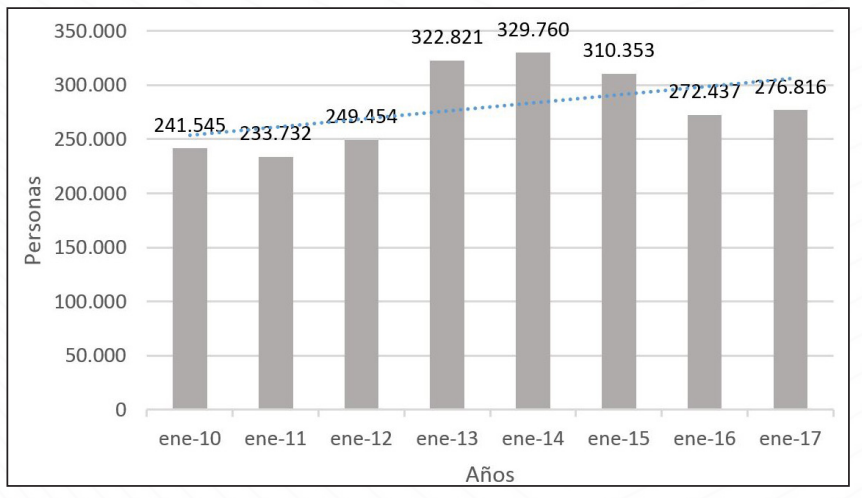

Fuente: Elaboración propia a partir del trabajo expuesto por el Instituto Nacional de Estadística y Censos INEC (2018) Figura 6. Sector de la construcción, empleo adecuado pleno

La tasa de empleo adecuado en el sector de la construcción de los periodos comprendidos entre el año 2010 y 2017, presenta una tendencia creciente, en el año 2013 y 2014 registra los más altos niveles, posteriormente en el año 2016 tuvieron una variación de -12,22\% en comparación con el año anterior y en el año 2017 presentó un crecimiento del 1,61\%, lo cual indica una recuperación de la industria después de presentar una tendencia marcada a la baja en los años 2014 y 2015.

Por otro lado, también se puede observar que a diciembre del año 2015 el empleo adecuado en el sector de la construcción era de 310.353 personas, en el año 2016 se tiene una variación de -5.161 personas, en el año 2017 el porcentaje de variación fue en menor medida, pero registrando una pequeña recuperación con una variación positiva de 22.442 personas es decir del 1,61\%. Como se puede evidenciar cuando se aprobó la Ley Orgánica para evitar la especulación sobre el valor de las tierras, en el año 2017 el sector tuvo una recuperación en el área de empleo adecuado.

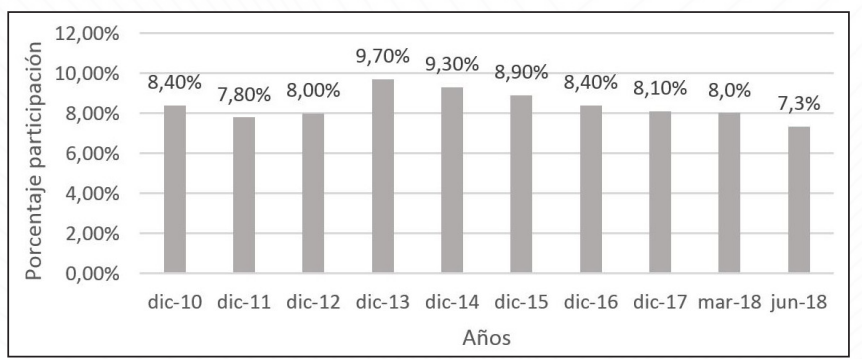

Fuente: Elaboración propia a partir del trabajo expuesto por el Instituto Nacional de Estadística Censos INEC (2018)

Figura 7. Participación del sector de la construcción en la Población Económicamente Activa

A través de la información que recolecta el Instituto Nacional de Estadística y Censos INEC sobre el mercado laboral ecuatoriano se obtiene que la participación del sector de la construcción en la Población Económicamente Activa PEA desde diciembre del año 2010 a diciembre del año 2017 ha sido del 8,4\%; 7,8\%; 8,0\%; 9,7\%; 9,3\%; $8,9 \% 8,4 \% ; 8,1 \%$ respectivamente, registrando una disminución desde el año 2014. 
Estos datos evidencian que el sector de la construcción ya presentaba decrementos desde el año 2014 lo cual indica una recesión económica que afectó principalmente en el año 2016 con una variación porcentual negativa, mayor que los periodos anteriores.

\section{Conclusiones}

Una vez validada la información proporcionada por el GAD Municipalidad de Ambato, se comprobó La Ley de Plusvalía es un factor que no tuvo mayor influencia por el lado contributivo debido al decrecimiento que ya se reflejaba en períodos anteriores, la recaudación resultante por efecto de las utilidades creció de $\$ 1.582 .432,22$ a $\$ 1.638 .577,55$, es decir existió un incremento de 3,5\%.

Se puede atribuir a la recesión en el sector a la recesión que vivió el país producto de la baja en el precio del petróleo, apreciación del dólar y medidas como las salvaguardas, además del terremoto de abril de 2016 en Manabí, el decrecimiento aparece desde 2016 donde se evidencia un decrecimiento de la inversión pública, es decir el tema impositivo no incidió en las tendencias negativas del sector de la construcción.

La información del sector financiero no se ha deteriorado relevantemente con excepción del periodo 2016, período en el cual no existió aplicación del llamado impuesto a la plusvalía, y más bien el país sufrió un proceso de recesión económica derivado de los factores que se han detallado anteriormente.

No ha existido una caída relevante para el periodo de vigencia del impuesto en lo que tiene que ver a la carga laboral, sin embargo la estadística negativa también se relaciona con la caída de la inversión pública.

Un tributo no puede ser analizado por su incidencia y efecto en apenas un año desde su creación, lamentablemente lo técnico se volvió político, el impuesto se eliminó a principios del 2018 y las condiciones del sector no han mejorado, una variable adicional que determina que el impuesto a la plusvalía no incidió en la rentabilidad del sector de la construcción.

Se determinó que existió un incremento de activos en el sector de la construcción en 2\%; sus pasivos se incrementaron en 3\%; y, su patrimonio decreció en -9\% correspondiente al año 2017, lo cual se permite apreciar que hubo una recuperación por el aumento de sus activos en comparación de años anteriores.

Las ventas del sector de la construcción presentan variaciones en los últimos períodos analizados, entre incremento y disminución, sin embargo, en el año 2016 y 2017 se reduce en $-27 \%$ y $-53 \%$ respectivamente.

Las ventas y las utilidades presentadas por el sector se evidencian resultados negativos en el año 2017 en donde se reducen drásticamente en comparación con el periodo anterior registrando inclusive pérdida de $\$ 385.528,47$ con una variación de $-141 \%$.

En años anteriores ya indicaba disminución en las ventas y utilidades, estas últimas se redujeron desde el año 2014, lo cual indica una recesión económica en el país, que junto con la desinformación y un análisis deficiente de la Ley de Plusvalía ocasionó que la inversión privada disminuyera. Los créditos hipotecarios que desde la publicación del nuevo proyecto de la Ley presentado en el año 2015, el sector ya tuvo una variación de $-10 \%$, de $-17 \%$ en el año 2016 y $-9 \%$ en el año 2017.

Se concluyó que los créditos hipotecarios otorgados al sector de la construcción, disminuyeron en el año 2016 de -16\%, en el 2017 con $-27 \%$. Así también las operaciones realizadas en el año 2016 registraron 22.140 operaciones y una variación de -17\%; en el año 2017 se registraron 20.218 operaciones con una variación de $-9 \%$.
Se determinó que la tasa de empleo adecuado en el sector de la construcción para el año 2017, presentó una variación positiva en relación del año anterior. Finalmente la participación del sector de la construcción en la Población Económica Activa, fue en decrecimiento desde el año 2014.

\section{Referencias}

Asamblea Nacional República del Ecuador. (2016). Ley Orgánica para evitarla especulación sobre el valor de las tierras y fijación de tributos. Quito: Registro Oficial Séptimo Suplemento. Obtenido de https://www. google. $\mathrm{com} /$ url?sa $=t \& r c t=j \& q=\& e s r c=s \&$ source $=$ web $\& c d=1 \& v e d=2 a-$ hUKEWillOSTZVTdAhXOoFMKHSA5BugQFjAAegQIBXAC\&url=http\%3A\%2F\%2Fwww.sri.gob.ec\%2FDocumentosAlfrescoPortlet\%2Fdescargar\%2F6a64a6c8-4512-4a00-ae96-0ab0c6511 Cd5\%2FLEY\%2BPUBLICADA\%2BEN\%2BEL\%2B

Camara de Comercio de Guayaquil. (2017). PIB 2016 y Proyecciones 2017. Obtenido de http://www.lacamara.org/website/ estadisticas/

Correa Delgado, R. (2017). Ley de Plusvalía. Economía en bicicleta, 7. Obtenido de http://economiaenbicicleta.com/ley-de-plusvalia/ Garza, N. F. (2005). Precios del suelo en Bogotá: crecimiento versus especulación. Territorios, 23. munidad, 23

Garza, R. (2017). Pega especulación a los bienes raíces. Co-

La Hora. (2016). La Ley de Plusvalía preocupa a varios sectores del país. Obtenido de https://lahora.com.ec/noticia/1102008728/ la-ley-de-plusvalc3ada-preocupa-a-varios-sectores-del-pac3ads

mundoconstructor.com.ec. (8 de julio de 2018). La inversión pública y privada cae y afecta al sector de la construcción. Obtenido de https://www.mundoconstructor.com.ec/la-inversion-publica-yprivada-cae-y-afecta-al-sector-de-la-construccion/

Quintana , E. (2010). ¿Por qué no son malos los especuladores? Economía y Negocios, 6.

Ramírez Carreño, S. (2016). Al 'boom' económico de Ecuador le llegó la recesión. Obtenido de https://www.eltiempo.com/archivo/ documento/CMS-16654045

Servicio de Rentas Internas. (2016). En qué difiere el impuesto vigente. Obtenido de http://www.sri.gob.ec/web/guest/ley-organica-especulacion-sobre-valor-de-las-tierras 